\title{
INTERLAYER EXCHANGE COUPLING: EFFECT OF ALLOYING
}

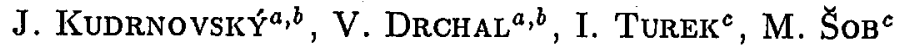 \\ AND P. WEINBERGER ${ }^{b}$ \\ ${ }^{a}$ Institute of Physics, Academy of Sciences of the Czech Republic \\ $\mathrm{Na}$ Slovance 2, 18040 Praha 8, Czech Republic \\ ${ }^{b}$ Institute for Technical Electrochemistry, Technical University of Vienna \\ Getreidemarkt 9, 1060 Vienna, Austria \\ 'Institute of Physics of Materials, Academy of Sciences of the Czech Republic \\ Žižkova 22, 61662 Brno, Czech Republic
}

The effect of disorder in magnetic layers, in the spacer, and at interfaces between them on periods, amplitudes, and phases of oscillations of interlayer exchange coupling in magnetic multilayers is studied theoretically on the $a b$ initio level. We employ the spin-polarized surface Green function technique within the tight-binding linear muffin-tin orbital method and the Lloyd formulation of the interlayer exchange coupling. The coherent potential approximation is used to describe the effect of alloying. The calculations are significantly simplified by employing the vertex-cancellation theorem. Numerical results illustrating the effect of various kinds of disorder in the system on the properties of the interlayer exchange coupling are presented.

PACS numbers: $75.70 . \mathrm{Cn}, 75.30 . \mathrm{Et}$

\section{Introduction}

The interlayer exchange coupling (IEC) between magnetic layers separated by a non-magnetic non-random spacer has recently been the subject of intense theoretical studies, particularly on the $a b$ initio [1-3]. The origin of the periods of oscillations of the IEC is now well understood. A new theoretical insight can be obtained by addressing the subtle problem of the variation of periods, amplitudes, and phases of the oscillations with the composition of magnetic and spacer alloy layers. Due to unavoidable interdiffusion, substitutional randomness is likely to occur at interfaces between magnetic and spacer layers. Thus, it is important to investigate the influence of disorder on the IEC.

It is the purpose of this paper to perform such a study on the ab initio level. We employ the Lloyd formulation of the IEC combined with a spin-polarized surface Green function technique as based on the tight-binding linear muffin-tin orbital (TB-LMTO) method [3]. The use of a Green function formulation of the IEC 
is essential for describing randomness within the coherent potential approximation (CPA) which is known to reproduce compositional trends in random alloys reliably. The calculations are significantly simplified by employing the vertex-cancellation theorem [4].

\section{Formalism}

Our model consists of two, generally different, semiinfinite magnetic subsystems denoted by $\mathcal{L}$ and $\mathcal{R}$, each containing $M$ magnetic layers on the top of a semiinfinite non-magnetic base and separated by a finite spacer slab $\mathcal{C}$ of varying thickness $N$. A special case is a model of two semiinfinite magnetic slabs sandwiching a finite spacer. Both magnetic and spacer layers are assumed to be binary alloys of arbitrary composition. We also assume a possible interface roughness or interdiffusion at the interfaces between magnetic and spacer layers. Further, $\vartheta$ is a relative angle between spin directions in the magnetic subsystems $\mathcal{L}$ and $\mathcal{R}$.

The exchange energy $\overline{\mathcal{E}}_{x}$ is defined as $\overline{\mathcal{E}}_{x}(\vartheta)=\bar{\Omega}(\vartheta)-\bar{\Omega}(0)$, where the overbar denotes the configurational averaging and $\Omega$ is the grand canonical potential. The concept of principal layers as used within the TB-LMTO method leads to a block tridiagonal form of the structure constants and of the inverse of the Green function $[2,3]$. Considering as a perturbation the interlayer coupling at the $\mathcal{L} / \mathcal{C}$ and the $\mathcal{C} / \mathcal{R}$ interfaces, which is independent of the thickness of the magnetic slabs, and employing the partitioning technique with respect to the trace of the logarithm of the Green function appearing in the expression for the grand canonical potential $[2,3]$, it is possible to extract directly the term describing the magnetic coupling of interfaces. The resulting expression for the configurationally averaged $\overline{\mathcal{E}}_{x}(\vartheta)$ is given by the following expression $[3,5]$ :

$$
\overline{\mathcal{E}}_{x}(\vartheta)=\frac{1}{\pi N_{\|}} \operatorname{Im} \sum_{k_{\|}} \int_{C} f(z) \operatorname{tr} \ln \left(1-\frac{1-\cos (\vartheta)}{2} M\left(k_{\|}, z\right)\right) \mathrm{d} z
$$

Here $f(z)$ is the Fermi-Dirac distribution function and tr denotes the trace over angular momentum indices. In Eq. (1) the energy integration is performed over a contour $C$ in the upper half of the complex energy plane, the sum runs over $k_{\|}$-vectors in the irreducible surface Brillouin zone, and $N_{\|}$is the corresponding unit surface area. The quantity $M\left(k_{\|}, z\right)$ is defined as*

$$
\begin{aligned}
M= & -\left(1-S_{10} \overline{\mathcal{G}}_{\mathcal{L}}^{\uparrow} S_{01} \overline{\mathcal{G}}_{\mathcal{R}}^{\uparrow}\right)^{-1} S_{10}\left(\overline{\mathcal{G}}_{\mathcal{L}}^{\uparrow}-\overline{\mathcal{G}}_{\mathcal{L}}^{\downarrow}\right) \\
& \times\left(1-S_{01} \overline{\mathcal{G}}_{\mathcal{R}}^{\downarrow} S_{10} \overline{\mathcal{G}}_{\mathcal{L}}^{\downarrow}\right)^{-1} S_{01}\left(\overline{\mathcal{G}}_{\mathcal{R}}^{\uparrow}-\overline{\mathcal{G}}_{\mathcal{R}}^{\downarrow}\right),
\end{aligned}
$$

where for matters of simplicity the arguments $k_{\|}$and $z$ have been omitted. The quantities $S_{01}\left(k_{\|}\right)$and $S_{10}\left(k_{\|}\right)$in Eq. (2) are the structure constants which couple neighboring (principal) layers, $\overline{\mathcal{G}}_{\mathcal{S}}^{\sigma}\left(k_{\|}, z\right), \mathcal{S}=\mathcal{L}, \mathcal{R}$, is the configurationally averaged surface Green function of the magnetic subsystem and $\sigma$ denotes the spin index $(\sigma=\uparrow, \downarrow)$. It should be noted that in the presence of randomness the expression for the IEC is formally analogous to the case of a non-random spacer [3] in the

*This form is equivalent to that given in Ref. [3] after substitution back into Eq. (1). It factorizes the spin-asymmetry and it is directly related to the RKKY-like theories. 
sense that the surface Green functions, which enter Eq. (2), are substituted by the corresponding configurational averages ${ }^{\dagger}$. The theoretical basis for this simplification, which reduces computational times by almost two orders of magnitude, relies on the "alloy force theorem" [6] and on the "vertex cancellation theorem" [4]. The latter theorem states that the vertex correction to the IEC due to randomness in the system is exactly zero. Strictly speaking, this theorem is valid only in the limit of infinitesimal rotations of the spin-quantization axis but the numerical studies [4] verified its validity even for the large angle $\vartheta$. This is very much in the spirit of the force theorem.

The present approach scales linearly with the thickness of both the magnetic slab and the spacer layer. This makes possible the efficient evaluation of the IEC for a large set of spacer thicknesses which, in turn, allows reliable extraction of periods, amplitudes and phases from calculated data. The theory determines periods, and in particular, amplitudes and phases of the oscillations from asymptotical expansions, i.e., for large spacer thicknesses. On the other hand, experimental data are usually available for small spacer thicknesses limited to regions with a possible preasymptotic behavior. The advantage of the present approach lies in the possibility to bridge both regions and thus verify the theoretical concepts employed in the analysis of experiments.

Periods, amplitudes, and phases of the oscillations are found from a discrete Fourier transform $F(q)$ performed on a large enough set of $N^{2} \overline{\mathcal{E}}_{x}(N)$ values. The periods of oscillations $p_{i}$ are then identified from the positions $q_{i}$ of pronounced peaks of $|F(q)|$ as $p_{i}=2 \pi / q_{i}$, their amplitudes $A_{i}$ as $A_{i}=(2 / n)\left|F\left(q_{i}\right)\right|$, where $n$ is the number of $N^{2} \overline{\mathcal{E}}_{x}(N)$ values used in the Fourier analysis, and the phases $\varphi_{i}$ from the relation $\varphi_{i}=\pi / 2-\arctan \left(\operatorname{Im} F\left(q_{i}\right) / \operatorname{Re} F\left(q_{i}\right)\right)$. Extensive numerical tests for model cases verified the reliability of this approach. In order to exclude preasymptotic behavior, the discrete Fourier transform is performed for values of $N^{2} \overline{\mathcal{E}}_{x}(N)$ corresponding typically to $N=20 \div 80$. The effect of temperature on the periods and phases of the oscillations was found to be negligible but it can obscure an analysis of the oscillation amplitudes $[3,7]$. Hence all calculations refer to $T=0 \mathrm{~K}^{\ddagger}$. Throughout this paper we will assume $\vartheta=\pi$.

\section{Particular systems}

For the reference system of $\mathrm{fcc}-\mathrm{Co} / \mathrm{Cu} / \mathrm{Co}(001)$ trilayers the following subjects will be discussed:

(i) The effect of interface roughness and interdiffusion on amplitudes of the oscillatory exchange coupling [8];

${ }^{\dagger}$ For the evaluation of the configurationally a veraged surface Green function in the present context we refer to the paper: J. Kudrnovský, I. Turek, V. Drchal, P. Weinberger, S.K. Bose, A. Pasturel, Phys. Rev. B 47, 16525 (1993) or to the recent review paper: J. Kudrnovský, I. Turek, V. Drchal, M. Sob, in: Stability of Materials, Eds. A. Gonis, P. Turchi, J. Kudrnovský, Plenum, New York 1996, p. 237.

$\$$ The efficiency of the present approach allows us to perform calculations with a large number of points in the irreducible part of the surface Brillouin zone (ISBZ) to obtain well-converged results even at $T=0 \mathrm{~K}$. In particular, we employ $8580 / 5100 / 2244 / 684$ points in the ISBZ for the first four energy points on a contour close to the Fermi energy and 144 points for the remaining 16 energy points on the contour. 
(ii) The effect of alloying in the spacer on periods and amplitudes of the oscillatory exchange coupling in metallic multilayers [5]; and

(iii) the dependence of amplitudes and phases of the oscillatory coupling on the composition of ferromagnetic layers [9]. For numerical details concerning the evaluation of the IEC 'we refer the reader to Ref. [3].

Most of the results discussed in this paper refer to the model of two semiinfinite magnetic slabs sandwiching a finite spacer. For this model the short-period oscillations (SPO) with the period of $p \approx 2.53$ monolayers (MLs) dominate [2,3] and this simplifies the theoretical analysis. In particular cases, however, the model of two $\mathrm{Co}(001)$ slabs each five monolayers thick separated by a spacer will be also considered, for which both the SPO and LPO (long-period oscillations) are present.

\subsection{Interface roughness and interdiffusion}

We wish to investigate the role of two general cases of imperfections frequently occurring at interfaces of artificially prepared materials like magnetic multilayers, namely:

(i) an interfacial roughness case modelled via randomly distributed large terraces of monolayer heights leading to fluctuations of the spacer thickness in both directions around an ideal interface [10] with the probability $r$, and

(ii) an interfacial interdiffusion case, in which magnetic and spacer atoms are mixed randomly with the probability $x$ at the system interfaces, i.e., instead of ideally sharp $\mathrm{Co} / \mathrm{Cu}$ interfaces we have two buried layers $\mathrm{Co}_{1-x} \mathrm{Cu}_{x} / \mathrm{Co}_{x} \mathrm{Cu}_{1-x}$ at each interface. The IEC for the interface roughness model defined above can be obtained from the following simple relation:

$$
\overline{\mathcal{E}}_{x}(N)=\sum_{n} w(N-n) \mathcal{E}_{x}(n)
$$

The deviations of the actual spacer thickness $n$ from its mean value $N$ are specified by the probabilities $w(N-n)$, where $w(0)=(1-2 r)^{2}+2 r^{2}, w( \pm 1)=$ $2 r(1-2 r), w( \pm 2)=r^{2}$, and $w(m)=0$ for $|m|>2$. The convolution-like form of the relation between $\mathcal{E}_{x}(n)$ and $\overline{\mathcal{E}}_{x}(N)$ implies that the amplitudes of oscillations with the wave number $q$ are reduced due to roughness by a factor $\tilde{w}(k)=$ $\left(1-4 r \sin ^{2}(k / 2)\right)^{2}$, namely the Fourier transform of the probabilities $w(n)$. An obvious consequence of this result is an efficient suppression of the amplitude of short period oscillations with the period close to $p=2 \mathrm{MLs}$, e.g., as for the case of the SPO for $\mathrm{Co} / \mathrm{Cu} / \mathrm{Co}(001)$ system. On the contrary, the LPO are influenced by interface roughness less efficiently.

The results of calculations for the case of two semiinfinite Co slabs are summarized in Table. The following conclusions can be drawn:

(i) The effect of interfacial roughness on the coupling strength of the SPO is quite large and the SPO are almost wiped out for $r=0.25$. It should be noted that the relative suppression of amplitudes of the SPO is not sensitive to the thickness of magnetic slabs (essentially the same relative suppression was found also for the SPO for two Co(001) slabs in fcc-Cu each five monolayers thick [8];

(ii) The effect of interfacial interdiffusion on the coupling strength is even more dramatic. An appreciable suppression of the amplitudes of the SPO is visi- 


\section{TABLE}

Relative amplitudes $A(\nu) / A(0)(\nu=r, x)$ of the short-period oscillations for two semiinfinite $\mathrm{Co}(001)$ subsystems sandwiching the $\mathrm{Cu}$-spacer. The values are given as a function of the interface roughness $r$ and the interface interdiffusion concentration $x$. The quantity $A(0)$ refers to the ideal Co/Cu-interface $(r=0.0$ or $x=0.0)$.

\begin{tabular}{l|c|l|c}
\hline \hline$r$ & $A(r) / A(0)$ & $x$ & $A(x) / A(0)$ \\
\hline 0.0 & 1.0 & 0.0 & 1.0 \\
0.05 & 0.66 & 0.02 & 0.69 \\
0.1 & 0.40 & 0.05 & 0.36 \\
0.25 & 0.01 & 0.1 & 0.12
\end{tabular}

ble already for $2 \%$ interdiffusion and amounts to an order of magnitude for $10 \%$ interdiffusion;

(iii) The fact that the strong suppression of the amplitude of the SPO does not depend on the Co slab thickness confirms the decisive role of interface region. We have further addressed this problem by performing calculations with $5 \%$ interdiffusion where one of interfaces was disordered while the other interface was kept ideal. The relative amplitude for the SPO in the latter case was 0.62 , i.e., almost two times larger in comparison with the value 0.36 given in Table.

The present study clearly demonstrates the high sensitivity of the coupling strength with respect to various kinds of interfacial imperfections and the decisive role of interface electron scattering for the exchange oscillatory coupling and, in turn, indirectly also for the giant magnetoresistance in metallic multilayers. The present study also shows that a detailed knowledge of the quality of the system interfaces is needed when comparing theoretical and experimental values of coupling strengths.

\subsection{Alloying in spacer layers}

The study of the effect of alloying in the spacer represents another powerful experimental and theoretical tool $[11-14]$ to verify existing models of the IEC by continuously varying the medium between the magnetic layers. We present numerical studies for an fcc-Cu spacer as alloyed with $\mathrm{Ni}$ or $\mathrm{Zn}$ or $\mathrm{Au}$. This choice is motivated by the fact that alloying with $\mathrm{Ni}$ or $\mathrm{Zn}$ alters the electron concentration. and, consequently, modifies the topology of the alloy Fermi surface which is closely related to the coupling periods in terms of Fermi surface spanning vectors [7]. The changes in the coupling periods can then be viewed as a contraction $(\mathrm{Ni})$ or the expansion (Zn) of the alloy Fermi surface. On the contrary, alloying of $\mathrm{Cu}$ with $\mathrm{Au}$ does not alter the electron concentration. In all cases, however, the coupling amplitudes are expected to be influenced by the presence of alloy disorder. Strictly speaking, in random alloys the Fermi surface is not well defined. In particular cases, however, when the alloy Fermi energy lies in the $s p$-part of the spectrum above the $d$-band complex, alloy broadening usually is weak causing therefore only little 

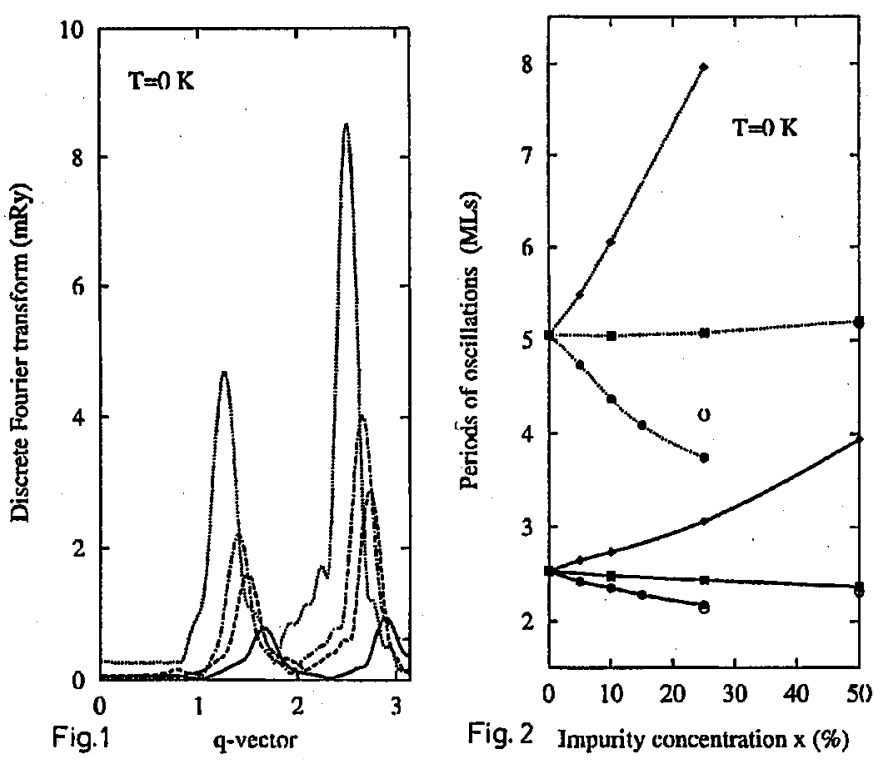

Fig. 2 Impurity concentration $x(\%)$

Fig. 1. Composition dependence of the absolute values of a discrete Fourier transform of $\overline{\mathcal{E}}_{x}$ at $T=0 \mathrm{~K}$ for two $\mathrm{Co}(001)$ slabs each five monolayers thick separated by a fcc- $\mathrm{Cu}_{1-x} \mathrm{Ni}_{x}$ alloy spacer: $\mathrm{Cu}_{0.75} \mathrm{Ni}_{0.25}$ (full line), $\mathrm{Cu}_{0.85} \mathrm{Ni}_{0.15}$ (dashed line), $\mathrm{Cu}_{0.9} \mathrm{Ni}_{0.1}$ (dashed-dotted line), and an ideal $\mathrm{Cu}$ spacer (dotted line).

Fig. 2. Composition dependence of the coupling periods at $T=0 \mathrm{~K}$ for two $\mathrm{Co}(001)$ slabs each five monolayers (MLs) thick separated by an $\mathrm{fcc}_{-} \mathrm{Cu}_{1-x} \mathrm{M}_{x}$ alloy spacer: $\mathrm{M}=\mathrm{Ni}$ (bullets), $\mathrm{M}=\mathrm{Au}$ (squares), and $\mathrm{M}=\mathrm{Zn}$ (diamonds). The lines serves as a guide for the eye and distinguish between short (full lines) and long (dotted lines) period oscillations. Open circles for $\mathrm{Cu}_{0.75} \mathrm{Ni}_{0.25}$ and $\mathrm{Cu}_{0.5} \mathrm{Au}_{0.5}$ represent the approximate virtual-crystal values. The periods are given in MLs.

smearing of the Fermi surface. As it is obvious from Eqs. (1-2), the present Green function formulation does not rely upon the existence of the alloy Fermi surface.

Alloying shifts corresponding peaks of $|F(q)|$ to higher values of $q$ and suppresses their heights as illustrated in Fig. 1 for the case of five MLs thick Co(001) slabs with an fcc- $\mathrm{Cu}_{1-x} \mathrm{Ni}_{x}$ spacer. The results for the concentration dependence of the periods of oscillations for the chosen alloy spacers are summarized in Fig. 2. For an ideal $\mathrm{Cu}$ spacer the SPO and the LPO are 2.53 MLs and 5.05 MLs, respectively. Alloying of $\mathrm{Cu}$ with $\mathrm{Ni}$ decreases the electron concentration in the alloy and leads to a contraction of the alloy Fermi surface. In terms of the RKKY-formulation [7] this leads to an expansion of spanning vectors corresponding to an fcc(001) layer orientation. Both the SPO and the LPO are reduced with increasing Ni-concentration but this reduction is more pronounced for the LPO. The present results for the LPO agree qualitatively with the simplified calculations of Ref. [12] based on a RKKY formulation and approximating the spanning vectors by a linear interpolation between the bulk band structures of $\mathrm{Cu}$ and $\mathrm{Ni}$. It is therefore interesting to compare the present results with those performed within the same computa- 
tional scheme but employing the VCA (virtual-crystal approximation) instead of the CPA. The results for $\mathrm{Cu}_{75} \mathrm{Ni}_{25}$ are shown in Fig. 2 as open circles. As one can see, in particular for the LPO the shift of the coupling periods differs in the VCA from that in the CPA.

The opposite behavior, namely a shift to larger periods and again rather pronounced for the LPO, was found when alloying $\mathrm{Cu}$ with $\mathrm{Zn}$, namely the case when the Fermi surface expands. Let us note that the periods of oscillations for a $\mathrm{Cu}_{1-x} \mathrm{Zn}_{x}$ spacer increase faster with $x$ than they decrease in the case of $\mathrm{Cu}_{1-x} \mathrm{Ni}_{x}$. Finally, for a $\mathrm{Cu}_{1-x} \mathrm{Au}_{x}$ spacer even for large concentrations of $\mathrm{Au}$ (up to $50 \%$ ), we observe a negligible concentration dependence of the periods of oscillations which is consistent with the composition-independent average electron number in these alloys as well as with available experimental data [13]. From Fig. 2 it is obvious that the coupling periods obtained for $\mathrm{Cu}_{0.5} \mathrm{Au}_{0.5}$ spacer by employing the CPA and the VCA are virtually the same. It should be noted that the results for the model of two semiinfinite Co slabs with the dominating SPO nearly coincides with those for the SPO in the present model.

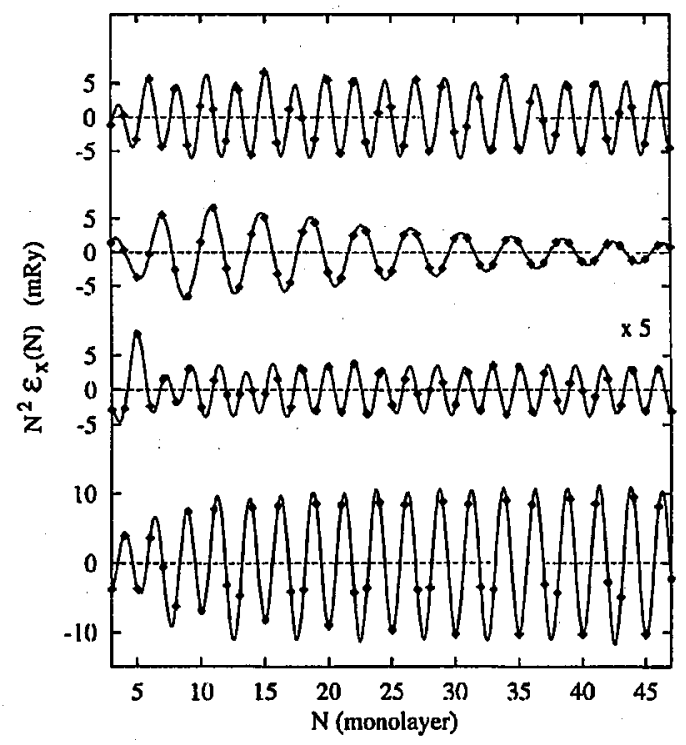

Fig. 3. Exchange coupling $N^{2} \overline{\mathcal{E}}_{x}(N)$ at $T=0 \mathrm{~K}$ as a function of the spacer thickness $N$ for two semiinfinite Co(001) subsystems sandwiching a spacer of (from bottom to top) ideal $\mathrm{Cu}, \mathrm{Cu}_{0.75} \mathrm{Ni}_{0.25}$ (multiplied by a factor 5), $\mathrm{Cu}_{0.5} \mathrm{Zn}_{0.5}$, and $\mathrm{Cu}_{0.5} \mathrm{Au}_{0.5}$. Diamonds refer to the calculated values, the full line (back Fourier transform) serves as a guide to the eye.

The dependence of $\overline{\mathcal{E}}_{x}$ on the spacer thickness $N$ is illustrated in Fig. 3 for the case of two semiinfinite $\mathrm{Co}(001)$ slabs sandwiching $\mathrm{Cu}_{0.75} \mathrm{Ni}_{0.25}, \mathrm{Cu}_{0.5} \mathrm{Zn}_{0.5}$, and $\mathrm{Cu}_{0.5} \mathrm{Au}_{0.5}$ alloy spacers. An increase in the coupling period is clearly seen for $\mathrm{Cu}_{0.5} \mathrm{Zn}_{0.5}$, while the reduction for a $\mathrm{Cu}_{0.75} \mathrm{Ni}_{0.25}$ spacer is less pronounced. The 
period of oscillations for $\mathrm{a} \mathrm{Cu}_{0.5} \mathrm{Au}_{0.5}$ alloy spacer remains essentially unchanged. From Fig. 3 one can also see that the $\mathrm{Cu}_{1-x} \mathrm{Ni}_{x}$ and $\mathrm{Cu}_{1-x} \mathrm{Au}_{x}$ alloy spacers exhibit a RKKY-like behavior with the amplitudes being proportional to $\mathrm{N}^{-2}$, however, with values reduced in comparison to the ideal $\mathrm{Cu}$ spacer. This reduction is particularly pronounced for a $\mathrm{Cu}_{0.75} \mathrm{Ni}_{0.25}$ spacer (see Fig. 1). An approximate RKKY-like behavior with a weak exponential damping $\propto \exp (-0.04 N)$ was found for $\mathrm{Cu}_{0.5} \mathrm{Zn}_{0.5}$.

The detailed understanding of the concentration dependence of amplitudes of oscillations is still missing. Adopting a RKKY-like picture, the SPO and the LPO periods are connected via so-called stationary points [7] located in different parts of the surface Brillouin zone. Quite likely, the different influence of alloying on the amplitudes of the SPO and the LPO [5] is connected with the anisotropy of electron-impurity scattering at the alloy Fermi level. Such information can be obtained, e.g., from surface-state resonance experiments [15].

\subsection{Alloying in magnetic layers}

Another insight into the physical nature of the IEC can be obtained by studying variation of the phases of oscillations with respect to the concentration in alloyed magnetic subsystems. A first experimental study of this kind for $\mathrm{fcc}(001)$ sputtered $\mathrm{Fe}-\mathrm{Co}-\mathrm{Ni} / \mathrm{Cu}$ multilayers [16] was significantly improved by preparing and measuring multiple MBE-grown sandwiches on the same single crystal substrate [17]. We consider symmetric systems, $A_{1-x} B_{x} / C u / A_{1-x} B_{x}(001)$, as well as asymmetric systems, $A_{1-x} B_{x} / C u / M(001)$ and $\mathrm{M}^{\prime} / \mathrm{Cu} / \mathrm{M}(001)$, where $\mathrm{A}_{1-x} \mathrm{~B}_{x}=\mathrm{Fe}_{1-x} \mathrm{Co}_{x}, \mathrm{Co}_{1-x} \mathrm{Ni}_{x}, \mathrm{Fe}_{1-x} \mathrm{Ni}_{x}$ and $\mathrm{M}, \mathrm{M}^{\prime}=\mathrm{Co}, \mathrm{Fe}$, Ni $\left(\mathrm{M} \neq \mathrm{M}^{\prime}\right)$. Alloying Co with $\mathrm{Ni}(\mathrm{Fe}$ ) increases (decreases) the average number of valence electron $N_{\mathrm{el}}$ of the ferromagnetic layers. As it is well known while studying $\mathrm{Fe}-\mathrm{Ni}$ alloys one can make use of the fact that certain compositions have the same $N_{\mathrm{el}}$ as in suitably chosen $\mathrm{Co}-\mathrm{Ni}$ or $\mathrm{Fe}-\mathrm{Co}$ systems. The role of a particular constituent as well as of $N_{\mathrm{el}}$ can be thus investigated. Finally, a study of asymmetric arrangements allows us to verify in detail predictions of a RKKY-like formulation of the IEC [7].

In Fig. 4 two symmetric alloyed magnetic layers, namely $\mathrm{Co}_{50} \mathrm{Ni}_{50}$ and $\mathrm{Fe}_{50} \mathrm{Co}_{50}$ layers, are compared with a reference case of ideal $\mathrm{Co}(001)$ layers. The results confirm qualitative predictions in terms of a RKKY-like formulation of the IEC: the dominant SPO of $2.53 \mathrm{MLs}$ is unaffected by alloying in the ferromagnetic layers [7]. The suppression of the amplitude of oscillations, pronounced in the $\mathrm{Co}_{50} \mathrm{Ni}_{50}$ case and rather weak for $\mathrm{Fe}_{50} \mathrm{Co}_{50}$ layers, is evident. The change of the phase of the oscillations due to alloying in the ferromagnetic layers can be also deduced from Fig. 4. In both the ideal and the alloyed case the so-called preasymptotic behavior is limited to the first 10 or 15 layers. This has to be kept in mind while making a detailed comparison with experiment, since experimentally the amplitudes and the phases are determined from the first antiferromagnetic peaks [17] rather than from the asymptotic behavior as in the present approach.

A summary of the concentration dependence of the phases of the oscillations for $\mathrm{Co}-\mathrm{Ni}, \mathrm{Fe}-\mathrm{Co}$, and $\mathrm{Fe}-\mathrm{Ni}$ layers is presented in Fig. 5. The following conclusions can be drawn: 


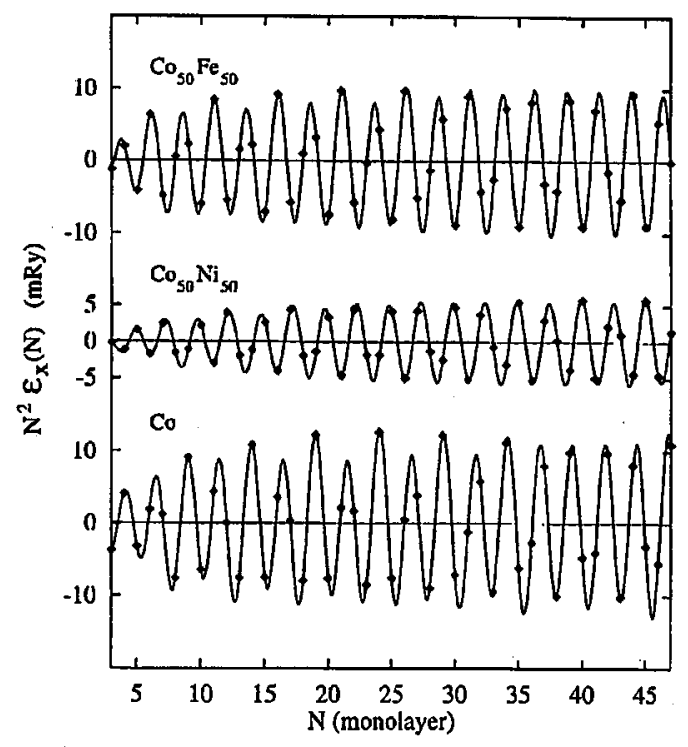

Fig. 4. Exchange coupling $N^{2} \overline{\mathcal{E}}_{x}(N)$ as a function of the spacer thickness $N$ for three different semiinfinite magnetic slabs separated by a fcc-Cu spacer. Diamonds refer to calculated values, the full lines (back Fourier transform) serve as a guide to the eye.

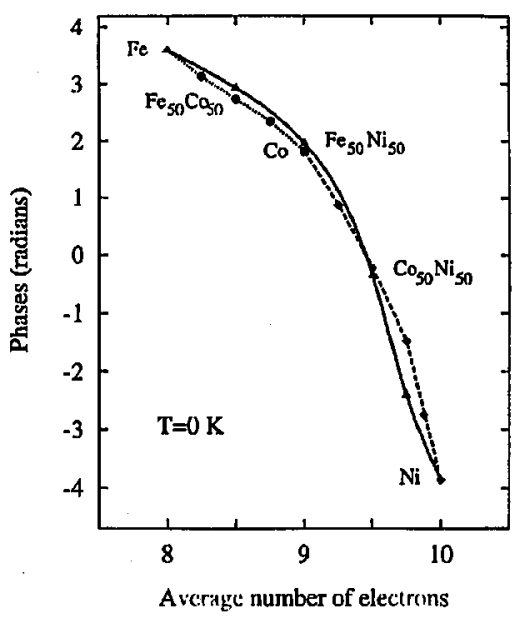

Fig. 5. Phases of the exchange coupling in semiinfinite magnetic slabs separated by a fcc- $\mathrm{Cn}$ spacer plotted as a function of the average number of valence electrons: $\mathrm{Fe}-\mathrm{Ni}(001)$ slabs (full line, triangles); $\mathrm{Fe}-\mathrm{Co}(001)$ slabs (dotted line, bullets), and $\mathrm{Co}-\mathrm{Ni}(001)$ slabs (dashed line, diamonds). The reference case of an ideal $\mathrm{Co}(001)$-slab is marked as a square. The lines serve as a guide to the eye. 
(i) in full agreement with experiments for fcc(001) faces [17] we observe a monotonic change of the phase with $N_{\mathrm{el}}$;

(ii) the estimated average phase variations of $0.56,0.63$, and $1.81 \pi$ /electron for $\mathrm{Fe}-\mathrm{Co}, \mathrm{Fe}-\mathrm{Ni}$, and $\mathrm{Co}-\mathrm{Ni}$ layers, respectively, should be compared to $0.65 \pi$ /electron found experimentally as an average from $\mathrm{Co}, \mathrm{Co}_{50} \mathrm{Ni}_{50}$, and $\mathrm{Fe}_{60} \mathrm{Ni}_{40}$ values [17]; and

(iii) for the same $N_{\mathrm{el}}$ the phases of the Fe-Ni layers agree very well with corresponding phases of $\mathrm{Co}-\mathrm{Ni}$ and $\mathrm{Fe}-\mathrm{Co}$ layers. The pronounced dependence of the phases on $N_{\mathrm{el}}$ and their insensitiveness to the specific elements which form the ferromagnetic layers is a relevant result of the present study. It can be explained qualitatively on the basis of a simplified RKKY-like formulation which relates (within a free electron picture) the amplitudes and phases to the wave vector of the spin-down transmitted wave in the ferromagnet, or, equivalently, to its electron density [7]. It should be noted that the alloys studied are strong ferromagnets with nearly filled spin-up bands. The changes of $N_{\mathrm{el}}$ caused by alloying are thus mostly at the cost of filling (emptying) of spin-down bands. Within the free electron picture the calculated opposite phase shifts for alloyed Co layers with elements having a higher $(\mathrm{Ni})$ or lower $(\mathrm{Fe})$ number of valence electrons as well as the observed insensitivity to the type of atoms which form ferromagnetic layers can be thus qualitatively understood. Deviations from the simple RKKY-picture reflect the differences between a realistic electronic structure and a free-electron model at the Fermi energy. The insensitivity of the phases of oscillations with respect to the atom type in the magnetic layers can further be supported by calculations for ternary $\mathrm{Fe}_{x} \mathrm{Co}_{y} \mathrm{Ni}_{z}$ magnetic layers, e.g., for the case $x=y=z=1 / 3$ with $N_{\text {el }}=9$. The calculated phase again compares very well with the corresponding one for Co layers with the same $N_{\mathrm{el}}$.

For one dominating period and large enough $N$ the IEC is given within a RKKY-like description [7] as

$$
\mathcal{E}_{x}(N) \approx \frac{1}{N^{2}} \operatorname{Im}[Z \exp (2 \pi \mathrm{i} q N)], \quad Z=Z_{1} Z_{2}, \quad Z_{i}=\sqrt{A_{i}} \exp \left(\mathrm{i} \varphi_{i}\right),
$$

where $Z_{i}(i=1,2)$ denote complex amplitudes characterizing magnetic subsystem 1 and $2(\mathcal{L}$ and $\mathcal{R})$. The wave vectors $q$ are identical with a relative accuracy $10^{-3}$ for all systems considered. We have used the values of the amplitudes $A^{\text {sym }}$ and the phases $\varphi^{\text {sym }}$ as calculated for symmetric systems $\mathrm{M} / \mathrm{Cu} / \mathrm{M}(001)$ and $\mathrm{A}_{1-x} \mathrm{~B}_{x} / \mathrm{Cu} / \mathrm{A}_{1-x} \mathrm{~B}_{x}(001)$ in order to find the complex amplitudes $Z_{i}^{\text {sym }}$ for the individual subsystems $A_{1-x} B_{x}$ and $M$ as

$$
Z_{i}^{\text {sym }}=\sqrt{A^{\text {sym }}} \exp \left(i \varphi^{\text {sym }} / 2\right) .
$$

Using these $Z_{i}^{\text {sym }}$ in Eq. (4) for a wide variety of asymmetric systems $\mathrm{A}_{1-x} \mathrm{~B}_{x} /$ $\mathrm{Cu} / \mathrm{M}(001)$ and $\mathrm{M}^{\prime} / \mathrm{Cu} / \mathrm{M}(001)$, we found quite a good agreement with the corresponding ab initio values of $Z$. A least squares fit applied to all data including symmetric and asymmetric systems yields values of $Z_{i}$ with a r.m.s. error not exceeding $5 \%$. This result represents perhaps the most complete confirmation of a RKKY-like approach to the IEC for the studied systems as most of the other existing comparisons concentrate only on the periods of oscillations. 
Financial support for this work was provided by the Austrian Science Foundation (project No. P10231), the Austrian Ministry of Science (Project No. GZ 45.384/2-IV/6/94), and the US-Czechoslovak Science and Technology program (project No. 95018).

\section{References}

[1] M. van Schilfgaarde, F. Herman, Phys. Rev. Lett. 71, 1923 (1993); P. Lang, L. Nordström, R. Zeller, P.H. Dederichs, Phys. Rev. Lett. 71, 1927 (1993); S. Krompiewski, U. Krey, J. Pirnay, J. Magn. Magn. Mater. 121, 238 (1993); S. Mirbt, H.L. Skriver, M. Aldén, B. Johansson, Solid State Commun. 88, 331 (1993).

[2] J. Kudrnovský, V. Drchal, I. Turek, P. Weinberger, Phys. Rev. B 50, 16105 (1994).

[3] V. Drchal, J. Kudrnovský, I. Turek, P. Weinberger, Phys. Rev. B 53, 15036 (1996).

[4] P. Bruno, J. Kudrnovský, V. Drchal, I. Turek, Phys. Rev. Lett. 76, 4254 (1996).

[5] J. Kudrnovský, V. Drchal, P. Bruno, I. Turek, P. Weinberger, Phys. Rev. B 54, R3738 (1996).

[6] F. Ducastelle, J. Phys. C, Solid State Phys. 8, 3297 (1975).

[7] P. Bruno, Phys. Rev. B 52, 411 (1995).

[8] J. Kudrnovský, V. Drchal, I. Turek, M. Šob, Phys. Rev. B 53, 5125 (1996).

[9] J. Kudrnovský, V. Drchal, R. Coehoorn, M. Sob, P. Weinberger, Phys. Rev. Lett. (submitted).

[10] Y. Wang, P.M. Levy, J.L. Fry, Phys. Rev. Lett. 65, 2732 (1990).

[11] S.N. Okuno, K. Inomata, Phys. Rev. Lett. 70, 1711 (1993); J.-F. Bobo, L. Hennet, M. Piecuch, J. Hubsch, J. Phys., Condens. Matter 6, 2689 (1994).

[12] S.S.P. Parkin, C. Chappert, F. Herman, Europhys. Lett. 24, 71 (1993).

[13] O. Leng, V. Cros, R. Schäfer, A. Fuss, P. Grünberg, W. Zinn, J. Magn. Magn. Mater. 126, 367 (1993).

[14] B. Lee, Y.-Ch. Chang, Phys. Rev. B 52, 3499 (1995).

[15] A.J. Baratta, A.C. Ehrlich, Phys. Rev. B 28, 4136 (1983); J. Grävenstein, I. Mertig, E. Mrosan, R. Zeller, J. Phys. F, Met. Phys. 18, 731 (1988).

[16] R. Coehoorn, J.P.W.B. Duchateau, J. Magn. Magn. Mater. 126, 390 (1993).

[17] M.T. Johnson, M.T.H. van de Vorst, P.J.H. Bloemen, R. Coehoorn, A. Reinders, J. van de Stegge, R. Jungblut, Phys. Rev. Lett. 75, 4686 (1995). 